\title{
A Criança Amada e Odiada: uma análise do status controverso
}

\author{
The Beloved and Hated Child: examining the controversial status
}

http://dx.doi.org/10.5007/2178-4582.2013v47n1p4

\author{
Bruna Camillo Bonassi, Mauro Luís Vieira, Ana Maria X. Faraco \\ Universidade Federal de Santa Catarina, Florianópolis/SC, Brasil \\ e Kenneth H. Rubin \\ University of Maryland, College Park/Maryland, USA
}

\begin{abstract}
$\mathrm{O}$ artigo discute uma pesquisa que teve dois objetivos: (a) caracterizar o status sociométrico controverso em crianças, diferenciando-o dos demais status; e (b) relacionar este status social à percepção da criança sobre suas relações com amigos e pais e à percepção dos professores sobre a criança controversa. Participaram do estudo 157 crianças (75 meninas) do 50 . ano do ensino fundamental, provenientes de duas escolas públicas e seis de seus professores. Os dados analisados derivaram de dois instrumentos 6 preenchidos pelos respondentes. Resultados indicaram que $10,2 \%$ da amostra era composta por crianças controversas e avaliadas por seus pares como altamente aceitas e altamente rejeitadas e, por seus professores, como crianças de baixo retraimento social e alta autonomia. As crianças controversas que se percebiam como alvo dos cuidados maternos também relataram maior intimidade com suas mães. Esses e outros resultados são discutidos considerando-se a qualidade dos relacionamentos sociais e do bem-estar psicológico da criança.
\end{abstract}

Palavras-chave: Criança controversa - Status sociométrico - Aceitação - Rejeição.
This article discusses a research carried out with two goals: (a) to characterize the controversial sociometric status in children differentiating it from other status; and (b) to relate this social status to the child's perception of their relationships with friends and parents and also to the teachers' perception on the controversial child. Participants were 157 children (75 girls) of two public schools, which were enrolled in 5th grade (elementary school), as well as 6 teachers of them. The data were derived from two instruments filled by participants. The findings have indicated that $10.2 \%$ of this sample was composed by controversial children, who were evaluated by their peers as highly accepted and highly rejected, and as low at social shyness and high at autonomy by their teachers. The controversial children who saw themselves as the focus of their mother's care have also reported greater intimacy with their mothers. These and other outcomes are discussed in this article, considering the quality of the social relationships and psychological well-being of children.

Keywords: Controversial child - Sociometric Status - Acceptance - Rejection.

\section{Introdução}

Muito se tem estudado sobre relações de amizade entre crianças e pré -adolescentes nas últimas quatro décadas. Sabe-se hoje que as relações de amizade da criança influenciam fortemente no desenvolvimento de diversas habilidades sociais e intelectuais infantis. Grupos de amigos ajudam a criança a desenvolver habilidades necessárias, como dar e receber ordens, participar 
de brincadeiras cooperativas, controlar a raiva, entre outros (FINE, 1987; RUBIN et al, 2005). A amizade também exerce influência na capacidade da criança de solucionar problemas, na sua competência social e ajustamento escolar (GIFFORD-SMITH; BROWNELL, 2003).

Além disso, crianças desenvolvem diferentes padrões de relacionamento com seus pares em função de suas experiências prévias com pais/cuidadores e irmãos (HINDE, 1987). Esses padrões de interação social tendem a ser estáveis e, portanto, podem influenciar a qualidade de suas amizades. Com o intuito identificar os diferentes padrões de comportamento da criança no grupo de pares, Coie, Dodge e Coppotelli (1982) delinearam cinco tipos de status sociométricos para crianças e pré-adolescentes: crianças populares, rejeitadas, negligenciadas, controversas e médias. Essa classificação tem por base nomeações positivas ou negativas dos pares sobre a criança, que constituirão índices de aceitação ou rejeição social dos padrões de comportamentos da criança. As crianças que não se encaixavam em nenhum destes grupos foram classificadas em "outros".

Assim, o status sociométrico deriva de uma avaliação dos pares sobre o comportamento social da criança, considerando as diferentes maneiras e habilidades que a criança demonstra ao estabelecer e conduzir suas interações e relações no grupo. Em geral, quando se trata de classificação sociométrica, o foco das pesquisas brasileiras e estrangeiras recai nos status que incluem maior quantidade de crianças: as rejeitadas e as populares. O status controverso costuma aparecer em último lugar recebendo, portanto, pouca atenção de pesquisadores. Daí a carência de informações precisas na literatura, assim como dados científicos sobre suas características e predominância.

\section{Caracterização do status controverso}

Para caracterizar o status controverso é necessário, primeiro, caracterizar brevemente os status sociométricos definidos por Coie et al (1982) por meio do Revised Class Play (MORISON; PELLEGRINI, 1985), um instrumento aplicado às crianças e composto de 35 itens, dos quais 2 indicadores de nomeação positiva e negativa - 'alguém de quem eu gosto de estar junto'; e, 'alguém de quem eu não gostaria de estar junto' - são utilizados, respectivamente, para compor os diferentes status.

Crianças populares: apresentam alto índice de nomeações positivas e baixo índice de nomeações negativas. Tendem a comportamentos pró-sociais e pouco agressivos. Segundo Coie e Dodge (1983), as crianças populares recebem dos seus colegas escores mais altos de cooperação, em comparação com outros grupos, e são vistas como líderes. Já nos aspectos negativos, costumam ter muito poucas indicações de comportamentos disruptivos e não são classificadas como crianças que começam brigas.

Crianças rejeitadas: apresentam alto índice de nomeações negativas e baixo índice de nomeações positivas. Tendem a apresentar comportamentos agressivos em sua maioria. São classificadas pelos colegas com escores 
baixos de cooperatividade, altos escores de comportamento disruptivos (tais como agressividade para com os colegas), como não líderes e como crianças que começam brigas (COIE et al, 1983).

Crianças negligenciadas: apresentam baixo nível de nomeações positivas e negativas, sendo crianças com pouca visibilidade na sala de aula. Tendem a apresentar comportamentos mais retraídos e níveis mais baixos de sociabilidade. São classificadas pelos colegas com altos escores de timidez (Ibid.).

Crianças controversas: apresentam alto índice de nomeações positivas e negativas, tendem a apresentar comportamentos de alta sociabilidade e alta agressividade. São classificadas pelos colegas com altos escores de cooperatividade, mas também de disruptividade e tendência a iniciar brigas (Ibid.).

Crianças médias: apresentam número médio de nomeações positivas e negativas. Ao contrário dos status extremos (populares, rejeitadas, negligenciadas e controversas) costumam compor o grupo mais frequente, ocupando cerca de metade da amostra.

Crianças mais pró-sociáveis e menos agressivas são preferidas pelos colegas para cargos de liderança e são consideradas crianças populares. Estas possuem redes mais amplas de relacionamento, percepções mais positivas do vínculo professor-aluno e são vistas pelos colegas como mais alegres (MARTINELLI; SCHIAVONI, 2009). Já as crianças agressivas, assim como as retraídas, tendem a ser mais excluídas e são vistas pelos colegas como crianças que perturbam ou se afastam do grupo. Tendem, assim, a ser classificadas como rejeitadas ou negligenciadas (ASCHER; COIE, 1990; NEWCOMB; BUKOWSKI; PATTEE, 1993). Além disso, possuem também uma visão mais negativa sobre sua interação com professores (MARTINELLI et al, 2009).

As crianças controversas apresentam características das rejeitadas e das populares ao mesmo tempo: recebem muitas nomeações positivas e negativas, com altos níveis de sociabilidade e agressividade (NEWCOMB et al, 1993). Essas crianças são descritas na literatura como membros altamente visíveis e identificáveis na sala de aula. Estudos indicam que, por serem muito chamativas e possuírem um caráter de liderança e agressividade bastante alto, é raro a coexistência de muitas crianças controversas por turma (MENESES, 2000).

As crianças controversas são percebidas pelos seus colegas como pessoas perturbadoras, que começam brigas e estão sempre pedindo ajuda nas tarefas escolares, antes mesmo de tentar resolvê-las ("seek help"), fator que foi associado com aprendizado mais lento (COIE et al, 1982). Porém, por serem socialmente ativas, e estarem quase sempre em atividades com pares e muito raramente isoladas, destacam-se como líderes de grupo. São caracterizadas como moderadamente cooperativas e com baixo nível de timidez, sendo vistas pelas outras crianças como alguém com quem se pode contar (COIE et al, 1982; MENESES, 2000). Segundo Newcomb et al (1993), os níveis de comportamento pró-social da criança controversa ultrapassam os níveis das crianças médias e populares. 
Crianças controversas também aparentam ter alto nível de controle sobre suas ações, podendo passar com facilidade de um comportamento agressivo para um pró-social, efeito denominado por Patterson (1993) como Quimera. Este efeito permite a modelagem e a melhor convivência com os pares, além de facilitar a relação com os professores, visto que elas adotam um comportamento pró-social quando estão sendo observadas. Devido ao efeito Quimera, o grupo de crianças descritas como controversas representa um desafio único para os educadores porque, embora elas tendam a se envolver, segundo o relato dos pares, em altas taxas de comportamento antissocial, essas crianças podem modelar seus comportamentos antissociais e pró-sociais e se engajar em comportamentos antissociais de forma encoberta, o que pode prejudicar ou incomodar seus pares, mas não atrai a atenção dos adultos (HILL; MERRELL, 2004). Por essa razão, essa habilidade torna difícil a observação e intervenção docente em comportamentos inadequados.

\section{Ocorrência do status sociométrico controverso em estudos brasileiros e estrangeiros}

De modo geral, a ocorrência do status sociométrico controverso é baixa nos estudos brasileiros, assim como nos estrangeiros. Bacalhau (2003) aponta que, num grupo de 73 crianças, apenas nove $(12,3 \%)$ foram classificadas como controversas. O estudo foi realizado em Lisboa, Portugal, com crianças de nove e dez anos, com predomínio do sexo feminino - 8 meninas e apenas 1 menino. Outro estudo, também realizado em Portugal, por Peceguina, Santos e Daniel (2008), com crianças de quatro anos de idade, aponta que de 74 sujeitos, apenas $4(5,4 \%)$ foram classificados como controversos. Como no estudo de Bacalhau (2003), este status teve baixa ocorrência.

Um estudo longitudinal realizado nos Estados Unidos na década de 1980 (COIE et al, 1983) já apontava o status controverso como o menos frequente. $\mathrm{Na}$ amostra analisada, verificou-se uma porcentagem de $5 \%$ em crianças de $3^{\text {a }}$. série do ensino fundamental e de $8 \%$ em crianças da $5^{\text {a }}$. série. Nesse estudo, os pesquisadores examinaram a estabilidade dos status sociométricos ao longo dos cinco anos, período em que o status controverso foi classificado como moderadamente estável, sendo o da criança rejeitada o mais estável. Um percentual de $31 \%$ das crianças consideradas controversas continuaram controversas após um ano; $24 \%$ em um intervalo de dois anos; $29 \%$ depois de três anos; e $14 \%$ depois de quatro anos. As crianças controversas tendem, segundo Coie et al (1983), a se tornarem rejeitadas num período de quatro anos, embora uma parcela significativa também migre para o status popular. É pouco provável, segundo os autores, que as crianças controversas sejam classificadas como crianças médias.

Estudo mais recente, também realizado nos Estados Unidos por Hill e Merrell (2004), mostrou que apenas 6,9\% das crianças foram apontadas como controversas por seus pais ou cuidadores, e $5,2 \%$ por seus professores. No estudo brasileiro de Pavarini, Loureiro e Souza (2009), foi observado um per- 
centual de 7,7\% de crianças controversas. Segundo Terry e Coie (1991), na maioria dos estudos, a proporção de crianças classificadas dentro dos status negligenciado, popular e rejeitado costuma variar entre 7\% e $15 \%$. O índice de crianças controversas fica aproximadamente entre $3 \%$ e $6 \%$, enquanto o status social médio representa $60 \%$ a $65 \%$ das crianças do grupo.

Considerando as implicações desse tema no processo de socialização e também a carência de estudos dessa natureza no Brasil, reforçam-se as razões e a necessidade de estudar a criança controversa. Portanto, a pesquisa de que trata este artigo teve como objetivo: (a) caracterizar o status controverso em crianças, diferenciando-o dos demais status, por meio de análises comparativas com outros grupos; e, (b) correlacionar este status social à percepção da criança sobre suas relações com amigos e pais e à percepção dos professores sobre a criança controversa.

\section{Método e instrumentos}

A amostra da pesquisa foi composta por 157 crianças que frequentavam a $4^{\text {a }}$. série do ensino fundamental ( 75 meninas, com idade média $=10,29$; DP $=0,803)$ e seis de seus professores, grupo proveniente de duas escolas públicas de Florianópolis, estado de Santa Catarina. Os dados analisados derivaram dos seguintes instrumentos: Revised Class Play (RCP) (MORISON et al, 1985); Network of Relationships Inventory (NRI) (FURMAN; BUHRMESTER, 1985); e a Escala Avaliativa da Criança (respondida pelo professor) criada por Hightower et al (1986).

O instrumento Revised Class Play acessou a reputação social da criança entre seus pares, por meio de perguntas que solicitavam à criança que se imaginassem diretores de uma peça de teatro e, como tal, indicassem quem, dentre seus colegas, melhor representaria determinados papeis, dentre uma lista entregue a cada criança. Para este estudo, foram usados dois itens da lista considerados como indicadores sociométricos. Por meio dos resultados padronizados das frequências absolutas dos itens 27 ('Alguém com que todos gostam de estar') e 33 ('Alguém com quem você não gostaria de estar'), foram criados os escores 'Like most' (Gosta mais) e 'Like least' (Gosta menos), a partir dos quais foram calculados os escores de Impacto Social (IS = LM + LL) e Preferência Social (PS = LM - LL).

A partir dos escores Impacto Social e Preferência Social foram calculados os cinco status sociométricos utilizados neste trabalho: crianças populares $-\mathrm{PS}>1.0, \mathrm{LM}>0$ e $\mathrm{LL}<0$; crianças rejeitadas $-\mathrm{PS}<1.0, \mathrm{LL}>0$ e $\mathrm{LM}<0$; crianças negligenciadas $-\mathrm{IS}<1.0$ e Like most $=0$; crianças controversas IS $>1.0$, LM e LL > 0; e crianças médias - PS e IS entre -0.5 e 0.5 (COIE et al, 1982; PECEGUINA et al, 2008).

Também foi realizada uma análise fatorial dos escores padronizados dos demais itens (papéis) do Revised Class Play, que resultou em quatro dimensões do funcionamento social da criança: Vitimização $(\propto=0,850)$; Sociabilidade $(\propto=0,942)$; Agressividade $(\propto=0,926)$; e Inibição $(\propto=0,740)$. A cada 
dimensão foram agregados os escores dos itens que a compunham, gerando assim um escore geral, em cada dimensão, para cada criança.

O instrumento Network of Relationships Inventory (NRI) contém 35 itens e acessou, separadamente, a qualidade do relacionamento da criança com três pessoas próximas e significantes (a mãe, o pai e o melhor amigo), em cada item, em uma escala de cinco pontos. Os participantes responderam de acordo com a sua percepção da qualidade da sua relação com cada uma dessas pessoas. Análises fatorais foram conduzidas para cada relacionamento e revelaram três dimensões em cada um deles. São elas: Cuidado-Mãe $(\propto=0,916)$; Relação afetiva proximidade-mãe $(\propto=0,833)$; Conflito-mãe $(\propto=0,830)$; Cuidado-pai $(\propto=0,887)$; Relação afetiva proximidade-pai $(\propto=0,879)$; Conflito-pai $(\propto=0,798)$; Companheirismo-amigo $(\propto=0,913)$; Relação afetiva próxima-amigo $(\propto=0,845)$; e Conflito-amigo $(\propto=0,802)$.

A Escala Avaliativa da Criança foi respondida pelo professor e acessou comportamentos elementares das crianças na escola, como comportamento social (e problemas relacionados) e competência acadêmica. Análises fatorais foram conduzidas e revelaram cinco dimensões: Autonomia/Liderança $(\propto=0,917)$; Sociabilidade/confiança $(\propto=0,889)$; Autocontrole $(\propto=0,889)$; Conduta de distúrbio $(\propto=0,926)$; Dificuldade Acadêmica $(\propto=0,916)$; e Retraimento Social $(\propto=0,772)$.

\section{Análise dos dados, resultados e discussão}

Para verificar se havia diferenças entre a qualidade das relações das crianças com as pessoas significantes próximas (pai, mãe e melhor amigo) foi realizado um Teste $t$ de amostras independentes entre o grupo das crianças controversas e o grupo das demais crianças. Os escores para este teste derivaram das dimensões do NRI. Foram também examinadas as diferenças entre crianças controversas e os demais grupos, relativas às variáveis obtidas do RCP, agressividade e sociabilidade. Finalmente, foi testada a diferença entre estes grupos do ponto de vista do professor, cujos escores foram obtidos na Escala Avaliativa da Criança respondida pelos professores.

Por meio de análise descritiva, observou-se que os cinco status sociométricos abarcaram $80,2 \%$ da amostra. Os percentuais dos status extremos (popular, rejeitada, negligenciada e controversa) foram: popular, 14\% (41\% meninos e $59 \%$ meninas); rejeitada, $23,6 \%$ (54\% meninos e $46 \%$ meninas); negligenciada, $0,6 \%$ (100\% meninos); e, controversa, $10,2 \%$ (50\% meninos e $50 \%$ meninas). As crianças médias apresentaram frequência de $31,8 \%$ (50\% meninos e $50 \%$ meninas).

Quanto ao gênero das crianças controversas, embora não tenha havido diferenças significantes nesta amostra, pesquisas sugerem que há um predomínio do sexo masculino neste status (BACALHAU, 2003; HILL; MERRELL, 2004). Tal argumento é proposto por Hill et al (2004), com base na alegação de que as meninas têm mais habilidade para controlar e manejar seus comportamentos diante de seus professores e pais, o que diminui o número de 
crianças do sexo feminino caracterizadas como controversas. Tendo em vista que a amostra utilizada neste estudo foi descrita pelos colegas de classe companheiros que podem ver as relações de seus colegas de uma forma mais horizontalizada e ampla (em certos aspectos) em comparação com o professor - é compreensível que a agressividade fique evidente também em relação ao sexo feminino, igualando a quantidade de crianças por sexo, visto que, com seus colegas, as meninas não sentem a necessidade de modelar tanto seus comportamentos como sentem face a seus professores.

\section{A caracterização da criança controversa segundo a amostra}

$\mathrm{Na}$ análise dos dados, constatou-se que a criança controversa foi percebida por seus colegas como agressiva e sociável (Tabela 1). Resultados semelhantes foram encontrados nas pesquisas de Coie et al $(1982 ; 1983)$, Newcomb et al (1993) e Meneses (2000). A presença de comportamentos antagônicos faz parte da definição da criança controversa e indica um caminho para entender seu comportamento pois, apesar da agressividade, tal como as crianças rejeitadas, ela consegue obter altos níveis de sociabilidade, o que demostra uma habilidade para relacionamentos sociais efetivos. Tal habilidade também está presente no estabelecimento e manutencão das amizades recíprocas. Na amostra estudada, evidenciou-se que em 93,75\% das crianças controversas houve reciprocidade na indicaçao de amigos, ou seja, as crianças citadas pelas controversas como melhores amigas/os também as citaram em primeiro ou segundo lugar como suas/seus melhores amigas/os. Esses dados vão ao encontro do que aponta a literatura já existente que, segundo Bacalhau (2003), indica que o grupo de crianças controversas é o segundo grupo com mais amizades recíprocas. Segundo Tortella (2001), as crianças populares compõem o grupo que mais apresenta amizades recíprocas.

Tabela 1. A percepção dos colegas sobre a criança controversa

\begin{tabular}{l|c|c|c}
\hline Questionário & Variável & $\begin{array}{c}\text { Nível de } \\
\text { significância }\end{array}$ & Médias dos escores \\
\hline & Sociabilidade & 0,000 & Controversa: 8,42 \\
$\begin{array}{l}\text { Revised } \\
\begin{array}{l}\text { Class Play } \\
\text { (RCP) }\end{array}\end{array}$ & Agressividade & 0,012 & Não controversa:-,96 \\
& & Controversa: 5,29 \\
\hline
\end{tabular}

Fonte: Tabela elaborada pelos autores 
Os dados obtidos nos questionários aplicados aos professores (Tabela 2) nos permitem constatar um resultado diferente dos dados obtidos pelos colegas de classe. As categorias Sociabilidade/Liderança e Condutas de Distúrbio não aparecem como variáveis significativas, o que pode revelar a alta habilidade de modulação do próprio comportamento, ou o efeito Quimera (Patterson, 1993), citado anteriormente. A variável Dificuldade Acadêmica também não é mencionada como significativa. Resultados semelhantes foram encontrados por Coie et al (1982) e Hill e Merrell (2004).

As variáveis apontadas pelos professores como significativamente diferentes dos outros grupos de crianças são a Autonomia/Liderança da criança controversa e seu baixo Retraimento Social. Tais resultados mostram que, na amostra estudada, as crianças controversas são vistas pelos seus professores como líderes, até mais do que as crianças populares, e menos inibidas também, o que demonstra um alto nível de sociabilidade e habilidades pró-sociais.

Curiosamente a agressividade, ou comportamentos disruptivos, característicos da criança controversa, não constam no relato dos professores, apenas no dos colegas. Este dado converge com estudos realizados por Newcomb et al (1993), em que tanto a sociabilidade quanto a agressividade da criança controversa não eram notadas da mesma forma pelos professores e pelos colegas de classe.

Tabela 2. A percepção do professor sobre a criança controversa

\begin{tabular}{l|c|c|c}
\hline Questionário & Variável & $\begin{array}{c}\text { Nível de } \\
\text { significância }\end{array}$ & Médias dos escores \\
\hline $\begin{array}{l}\text { Escala } \\
\text { avaliativa do } \\
\text { professor }\end{array}$ & $\begin{array}{c}\text { Autonomia- } \\
\text { Liderança }\end{array}$ & 0,032 & Controversa: 39,71 \\
& $\begin{array}{c}\text { Retraimento } \\
\text { social }\end{array}$ & 0,007 & Não Controversa: 35,45 \\
Controversa: 6,71 \\
\hline
\end{tabular}

Fonte: Tabela elaborada pelos autores

Os dados obtidos por meio do questionário NRI (que acessa, separadamente, a relação da criança com três membros próximos: o pai, a mãe e o amigo) foram significativos somente quando referentes à mãe (Tabela 3 ). Esse é um dado importante e novo, uma vez que pesquisas que fazem referência ao papel materno na conduta da criança controversa são escassas, o que dificulta o contraste destes resultados com o de outros estudos. Os resultados indicaram, portanto, que as crianças controversas participantes da amostra apresentaram tendência a se perceberem mais próximas de suas mães, numa relação 
de confiança e intimidade com elas, do que as crianças dos outros grupos, percebendo-se como alvos de maiores cuidados por parte de suas mães. Isso pode indicar que, por se perceberem mais íntimas e mais cuidadas, se sintam mais seguras com relação às suas mães, o que possibilita a abertura para o mundo de forma também mais segura, reforçando a sociabilidade como característica dessas crianças. Assim, supõe-se que, por serem mais sociáveis, expressivas e por não passarem despercebidas, a relação de proximidade com as mães, por meio de um mecanismo de feedback, fique ainda mais facilitada (NEWCOMB, et al, 1993).

Tabela 3. A percepção da criança sobre sua reação com sua mãe ou cuidadora

\begin{tabular}{llll}
\hline Questionário & Variável & $\begin{array}{c}\text { Nível de } \\
\text { significância }\end{array}$ & Médias dos escores \\
\hline
\end{tabular}

Controversa: 78,56

$$
\text { Cuidado Mãe } \quad 0,047
$$

Network

Relationship

Inventory

(NRI)

$$
\begin{aligned}
& \text { Relação afetiva } \\
& \text { próxima - mãe }
\end{aligned} \quad 0,088
$$

Não controversa: 74,67

Controversa: 45,81

Não controversa: 41,98

Fonte: Tabela elaborada pelos autores

Como se constata, o status controverso continua sendo, nesta amostra, um status de baixa frequência, embora na comparação com estudos estrangeiros tenha apresentado frequência mais elevada $(10,2 \%)$. O grupo controverso apareceu como penúltimo no conjunto do demais grupos de crianças, à frente do status negligenciado. Essa estatística diverge do estudo realizado por Terry et al (1991) no qual o status controverso, de menor frequência, obteve em média uma porcentagem de $3 \%$ a $6 \%$. Outro estudo brasileiro que foge a esta estatística foi aquele realizado por Pavarini et al (2009), em que o percentual de crianças controversas foi de $7,7 \%$.

Vale ressaltar ainda que foi observada a presença relativamente maior de crianças controversas (quando comparado a outros estudos) em relação à quantidade média de crianças por turma $(3,2 \%$ controversas) da amostra. Esses dados trazem à tona a questão de por que tantas crianças fazem parte do status controverso. Sugere-se que futuras pesquisas levem em conta este fator no estudo desse status com vistas a buscar possíveis explicações para estes aspectos específicos. 


\section{Conclusão}

Os resultados desta pesquisa fortalecem a necessidade de estudos mais aprofundados do status controverso, pois a partir desse conhecimento será possível pensar práticas tanto parentais quanto escolares que possam melhorar a qualidade de vida das crianças classificadas nesse grupo e auxiliá-las a regular e controlar sua agressividade e/ou comportamentos impulsivos, de forma a melhorar suas interações com outras crianças.

O estudo do efeito Quimera, por exemplo, pode trazer uma compreensão muito maior da dinâmica desses comportamentos em sala de aula. Entender o porquê de a criança controversa ser muito aceita em determinado momento e rejeitada em outro é um desafio que demonstra a complexidade deste status. Por exemplo, se há, na criança controversa, a habilidade de modular seu comportamento de forma a parecer mais sociável frente a seus professores, por que então essa habilidade não é utilizada com seus colegas?

As práticas parentais também são uma fonte de informação preciosa sobre o comportamento da criança controversa, mas ainda muito pouco estudada. O reduzido número de pesquisas sobre crianças de perfil "controverso" e sua interação com professores, colegas e pais é um tópico ainda a ser explorado e que merece mais pesquisas na área. Isto porque o exame dos fatores correlatos, concomitantes e contextuais do status controverso, pode trazer novos insights sobre desenvolvimento, habilidades e dificuldades nas relações sociais de crianças desse grupo, bem como oferecer subsídios para propor intervenções mais eficientes tanto no âmbito escolar como familiar.

\section{Referências bibliográficas}

ASCHER, Steven R.; COIE, John. D. Peer Rejection in Childhood.Cambridge: University of Cambridge Press, 1990.

BACALHAU, Ana I. dos S. Amizade, reciprocidade e qualidade: A relação existe? Lisboa, 2003. Tese (Doutorado em Psicologia Educacional). Instituto Universitário de Ciências Psicológicas Sociais e da Vida, ISPA, Lisboa, 2003.

COIE, John D.; DODGE, Kenneth A.; COPPOTELLI, Heide. Dimensions and types of social status: A cross-age perspective. Developmental Psychology, [S.1.], v. 18, n. 4, p. 557-570, jul 1982.

COIE, John. D.; DODGE, Kenneth. A. Continuities and changes in children's social status: Afive-year longitudinal study. Merril-Palmer Quarterly, [S.1.], v. 29, n. 3, p. 261-282, jul 1983.

FINE, Gary. A. With the boys: Little league baseball and preadolescent culture. Chicago: University of Chicago, 1987.

FURMAN, Wyndol.; BUHRMESTER, Duane. Children's perceptions of the personal relationships in their social networks. Developmental Psychology,[S.1.], v. 26, n.6, p. 1016-1024, nov 1985. 
GIFFORD-SMITH, Mary. E.; BROWNELL, Celia. A. Childhood peer relationships: social acceptance, friendships, and peer networks. Journal of School Psychology, [S.1.], v. 41, n. 4, p. 235-284, jul/ago 2003.

HIGHTOWER, A. Dirk et al The Teacher-Child Rating Scale: A brief objective measure of elementary children's school problem behaviors and competencies. School Psychology Review, [S.1.], v. 15, n. 3, p. 393-409, jul 1986.

HILL, Diane K.; MERRELL, Kenneth. W. Characteristcs of "controversial" children: an exploration of teacher and parent social behavior rating scale datasets. Psychology in the Schools, [S.1.], v. 41, n. 5, p. 497-507, mar 2004.

HINDE, Robert A. Individuals, relationships and culture: Links between ethology and the social sciences. New York: Cambridge University Press, 1987.

MARTINELLI, Selma de C.; SCHIAVONI, Andreza. Percepção do aluno sobre sua interação com o professor e status sociométrico. Estudos de Psicologia, Natal, RN, v. 26, n. 3, p. 327-336, jul/set 2009.

MENESES, Helena I. M. A competência social no contexto escolar: Um estudo entre a competência social e a realização acadêmica. Porto, Portugal, 2000. Dissertação (Mestrado em Psicologia) Faculdade de Psicologia e Ciências da Educação, Universidade do Porto, Porto, Portugal, 2000 .

MASTEN, Ann. S.; MORISON, Patricia; PELLEGRINI, David S. A revised class play me $\neg-$ thod of peer assessment. Developmental Psychology, [S.1.],v. 21, n. 3, p. 523-533, mai 1985.

NEWCOMB, Andrew. F.; BUKOWSKI, Willian. M.; PATTEE, Linda. Children's peer rela $\neg-$ tions: A meta-analytic review of popular, rejected, neglected, controversial, and average sociometric status. Psychological Bulletin, [S.1.], v. 113, n. 1, p. 99-128, jan 1993.

PATTERSON, Gerald R.. Orderly change in a stable world: The antisocial trait as chimera. Journal of Consulting and Clinical Psychology,[S.1.], v. 61, n. 6, p. 911-919, dez 1993.

PAVARINI, Gabriela; LOUREIRO, Carolina P.; SOUZA, Débora D. Compreensão de emoções, aceitação social e avaliação de atributos comportamentais em crianças escolares. Psicologia: Reflexão e Crítica, Porto Alegre, RS, v. 24, n. 1, p. 135-143, out/dez 2009.

PECEGUINA, Inês; SANTOS, Antônio. J.; DANIEL, João. R. A concordância entre medidas sociométricas e a estabilidade dos estatutos sociais em crianças de idade pré-escolar. Análise Psicológica, Lisboa, v. 26, n. 3, p. 479-490, jul/ago 2008.

RUBIN, Kenneth H. et al. A. Peer relationships in childhood. In: BORNSTEIN, M. H.; LAMB, M.E. Social and Emotional Development: An Advanced Textbook. New York: Psychology Press, 2005.

TERRY, Robert; COIE, John. A comparison of methods for defining sociometric status among children. Developmental Psychology, [S.1.],v. 27, n. 5, p. 867-880, set 1991.

TORTELlA, Jussara C. B. A representação da amizade em díades de amigos e não amigos. Campinas, SP, 2001. Tese (Doutorado em Educação) Faculdade de Educação, Universidade Estadual de Campinas, Campinas, SP, 2001.

Recebido em: 11/12/2012

Aceite em: 23/08/2013 
Bruna Camillo Bonassi é estudante da graduação em Psicologia da UFSC. Endereço para correspondência: Núcleo de Estudos e Pesquisas em Desenvolvimento Infantil, Campus Universitário, Trindade. Florianópolis/SC, Brasil. CEP 88040-900 E-mail: bruna.bonassi@gmail.com

Mauro Luís Vieira é Professor Doutor do Departamento de Psicologia UFSC. E-mail: maurolvieira@gmail.com

Ana Maria X. Faraco é Professora Doutora colaboradora voluntária do Departamento de Psicologia da UFSC. E-mail: anna_marbr@yahoo.com.br

Kenneth H. Rubin é Professor Ph.D da University of Maryland, College Park, Maryland, USA. E-mail: krubin@umd.edu 